\title{
THE SPACE OF RETRACTIONS OF A 2-MANIFOLD
}

\author{
NEAL R. WAGNER
}

\begin{abstract}
Let $M^{2}$ be a 2-manifold and let $\Lambda$ be the embedding of $M^{2}$ into its space of retractions which maps each point to the constant retraction to that point. Denote by $\mathscr{L}\left(M^{2}\right)$ the component containing the image of $\Lambda$. The embedding $\Lambda$, with range restricted to $\mathscr{L}\left(M^{2}\right)$, is shown to be a weak homotopy equivalence if $M^{2}$ is compact, or if $M^{2}$ is complete and the metric topology is used.
\end{abstract}

1. Introduction. In [8], the author studied one component of the space of retractions of the 2-sphere and the annulus, namely the component consisting of retractions with contractible image (the nullhomotopic retractions). This paper extends the results of [8] to more general 2-manifolds. The techniques are similar to those in [8], and we refer the reader to that paper. The author would like to express his gratitude to the referee of [8] for suggesting the method of proof in this paper and to C. W. Neville for some helpful conversations.

For any 2-manifold $M^{2}$, let $\mathscr{R}\left(M^{2}\right)$ denote the space of retractions of $M^{2}$, with either the compact-open or the sup-metric topology. Let $\Lambda$ be the embedding of $M^{2}$ into $\mathscr{R}\left(M^{2}\right)$ which takes each $u \in M^{2}$ to the constant retraction of $M^{2}$ to $u$. Denote by $\mathscr{L}\left(M^{2}\right)$ the component of $\mathscr{R}\left(M^{2}\right)$ containing the image of $\Lambda$. We can now state the main result of this paper.

THEOREM. The embedding $\Lambda: M^{2} \rightarrow \mathscr{L}\left(M^{2}\right)$ is a weak homotopy equivalence in two cases:

(1) if $M^{2}$ is compact and the compact-open (=sup-metric) topology is used, or

(2) if $M^{2}$ is complete and the sup-metric topology is used.

These cases are exactly those for which $\mathscr{L}\left(M^{2}\right)$ consists of all retractions with compact, contractible image (see $\S 3$ ). Any (second countable)

Presented to the Society, May 17, 1971; received by the editors August 16, 1971.

AMS 1970 subject classifications. Primary 54C15, 54C35, 57A05; Secondary 54C65, 55D10, 30A 30.

Key words and phrases. Retract, retraction, two-manifold, weak homotopy equivalence, function space, compact-open topology, sup-metric, selection, Riemann surface, conformal mapping, zero-regular convergence.

(c) American Mathematical Society 1972 
2-manifold is complete in some metric, so case (2) of the theorem will apply in general with respect to some sup-metric on $\mathscr{R}\left(M^{2}\right)$.

By definition [7, p. 404], $\Lambda$ is a weak homotopy equivalence if the induced maps $\Lambda_{*}: \pi_{n}\left(M^{2}\right) \rightarrow \pi_{n}\left(\mathscr{L}\left(M^{2}\right)\right)$ are isomorphisms, for each $n$. If ev denotes the evaluation map $\left(\operatorname{ev}(\varphi)=\varphi\left(u_{0}\right)\right.$ for any retraction $\varphi$ and some basepoint $u_{0}$ of $M^{2}$ ), then clearly ev $\circ \Lambda$ is the identity map on $M^{2}$, so that it will suffice to prove that $\Lambda_{*}$ is surjective. The underlying idea of the proof is to produce, corresponding to certain retractions $\varphi$, a canonical simple closed curve in $M^{2}$ which bounds a disk containing the image of $\varphi$. Then, essentially working within the disk, we can construct a homotopy from $\varphi$ to $\Lambda \circ \operatorname{ev}(\varphi)$. These simple closed curves will be provided by a selection theorem of $\mathrm{E}$. Michael. A finite-dimensionality condition in this theorem prevents us from simply showing that $\Lambda \circ \mathrm{ev}$ is homotopic to the identity map on $\mathscr{L}\left(M^{2}\right)$ and concluding that $\Lambda$ is a homotopy equivalence.

2. Preliminaries on conformal mapping. In any 2-manifold $M^{2}$, let $\mathscr{S}$ denote the collection of simple closed curves which bound a disk in $M^{2}$ and which do not meet $\partial M^{2}$. (Since the theorem was proved in [8] for $S^{2}$, we assume $M^{2}$ is not $S^{2}$.) We shall use much of the notation of [8], which included $E^{2}$ for Euclidean 2-space, $B^{2}$ for the unit 2-ball, and $C_{r}$ for the circle with center at the origin and radius $r>0$. In addition, we denote the closed disk which $J \in \mathscr{S}$ bounds by $B(J)$, and its interior by $\operatorname{int}(B(J))$. If $K \in \mathscr{S}$ and $J \subset \operatorname{int}(B(K))$, then let $A(J, K)$ denote the closed annular region bounded by $J$ and $K$. In particular, we use $A^{2}$ for $A\left(C_{1}, C_{2}\right)$.

The topology of 0 -regular convergence can be defined on $\mathscr{S}$ as follows: a sequence $\left\{J_{i}\right\}$ converges 0 -regularly to $J_{0}$ if there are embeddings $f_{i}: C_{1} \rightarrow M^{2}$ such that $f_{i}\left(C_{1}\right)=J_{i}$ and $\left\{f_{i}\right\}$ converges uniformly to $f_{0}$ on $C_{1}$. (It can be seen from the work helow that on $\mathscr{S}$ this definition is equivalent to the usual one. See [3] and [4].)

LEMMA 1. Let $F$ be any compact, contractible subset of $M^{2}$, not meeting $\partial M^{2}$. Then there is a $J \in \mathscr{S}$ such that $F$ is contained in $\operatorname{int}(B(J))$.

Proof. The universal covering space of $M^{2} \backslash \partial M^{2}$ will be the plane $E^{2}$ or the 2-sphere $S^{2}[1, \mathrm{p}$. 104]. In either case, $F$ lifts to a homeomorphic copy $\hat{F}\left[7\right.$, p. 66], and $E^{2} \backslash \hat{F}$ is homeomorphic to $E^{2}$ minus a point (and likewise for $S^{2}$ ), so it is easy to produce a family of simple closed curves about $\hat{F}$. One of these which is close enough to $\hat{F}$ will project to a suitable curve about $F$ in $M^{2}$, completing the proof.

Let $M^{2}$ be any orientable 2-manifold. Give $M^{2}$ a conformal structure so as to make it a Riemann surface. (See [1] and [6].) Suppose that for each nonnegative integer $i$, elements $J_{i}$ and $K_{i}$ of $\mathscr{S}$ are given such that each $J_{i}$ lies in $\operatorname{int}\left(B\left(K_{i}\right)\right)$. Then there are homeomorphisms $f_{i}: B^{2} \rightarrow B\left(J_{i}\right)$ and 
$g_{i}: A^{2} \rightarrow A\left(J_{i}, K_{i}\right)$ which are uniquely determined by the conditions:

$\left(\mathrm{a}_{1}\right)$ each $f_{i}$ is conformal on $\operatorname{int}\left(B^{2}\right)$,

$\left(\mathrm{a}_{2}\right)$ each $f_{i}$ maps the point $(1,0)$ to some $v_{i} \in J_{i}$,

$\left(\mathrm{a}_{3}\right)$ each $f_{i}$ maps the origin to some $u_{i} \in \operatorname{int}\left(B\left(J_{i}\right)\right)$,

$\left(\mathrm{b}_{1}\right)$ each $g_{i}$ is a radial contraction of a map conformal on $\operatorname{int}\left(A\left(C_{1}, C_{r(i)}\right)\right)$, for some $r(i)>1$, and

$\left(\mathrm{b}_{2}\right)$ each $g_{i}$ maps $(1,0)$ to $v_{i}$.

Moreover, we have the following result.

Continuity Property. If $\left\{J_{i}\right\}$ and $\left\{K_{i}\right\}$ converge 0-regularly to $J_{0}$ and $K_{0}$, respectively, and if $\left\{u_{i}\right\}$ and $\left\{v_{i}\right\}$ converge to $u_{0}$ and $v_{0}$, respectively, then $\left\{f_{i}\right\}$ converges uniformly to $f_{0}$ on $B^{2}$ and $\left\{g_{i}\right\}$ converges uniformly to $g_{0}$ on $A^{2}$.

Proof. Select $K \in \mathscr{S}$ such that $K_{i}$ is contained in $\operatorname{int}(B(K))$ for all sufficiently large $i$. The set int $(B(K))$ is an open simply connected Riemann surface, clearly of hyperbolic type, and hence $([1$, p. 203], [6, p. 197]) is conformally equivalent to int $\left(B^{2}\right)$. This translates the situation to the plane, where the results are known. (See [3], [4], and [8].)

If $M^{2}$ is not orientable, it has a conformal structure provided conjugates of conformal maps are included. The above results will still hold if all the maps involved are conformal with respect to an orientation of the domain $\operatorname{int}(B(K))$.

3. Proof of the theorem. The proof uses a selection theorem of E. Michael [5], which we state here in a very weak form.

THEOREM M. Let $B$ and $X$ be metric spaces, where $B$ is complete and the dimension of $X$ is $\leqq n$. Let $\tau$ be an open mapping of $B$ onto $X$ such that each (point) inverse under $\tau$ has vanishing homotopy groups of order $\leqq n-1$, and such that the collection of inverses under $\tau$ is equi-LC $C^{n-1}$. Let e be a mapping of a closed subspace $Y$ of $X$ into $B$ such that $e(y) \in \tau^{-1}(y)$, for all $y \in Y$. Then e extends to a mapping $e^{*}$ of $X$ into $B$ such that $e^{*}(x) \in \tau^{-1}(x)$, for all $x \in X$.

(The property equi-LC $\mathrm{L}^{n-1}$ is a strong form of $\mathrm{LC}^{n-1}$. See [5] and [8, Definition 1.4].)

Let $M^{2}$ be any 2-manifold, and denote by $\mathscr{K}\left(M^{2}\right)$ the space of those retractions of $M^{2}$ with compact, contractible image. The construction in this section is designed to handle $\mathscr{K}\left(M^{2}\right)$, and it will follow from later work that $\mathscr{K}\left(M^{2}\right)$ is pathwise connected. If $M^{2}$ is compact, there is a number $\varepsilon$ such that any two self maps within a distance $\varepsilon$ are homotopic. Since the elements of $\mathscr{K}\left(M^{2}\right)$ are nullhomotopic, it is clear that $\mathscr{L}\left(M^{2}\right)=\mathscr{K}\left(M^{2}\right)$. If $M^{2}$ is complete and we use the sup-metric topology, then for any $\varphi \in \mathscr{K}\left(M^{2}\right)$, we can produce a compact submanifold $N^{2}$ of $M^{2}$ and an 
$\varepsilon>0$ such that $d(\psi, \varphi)<\varepsilon$ implies $\operatorname{im}(\psi) \subset N^{2}$, and so for some $\varepsilon^{\prime}>0$, $d(\psi, \varphi)<\varepsilon^{\prime}$ implies $\psi \in \mathscr{K}\left(M^{2}\right)$, i.e., $\mathscr{K}\left(M^{2}\right)$ is open in $\mathscr{R}\left(M^{2}\right)$. (We can construct $N^{2}$ by covering $\operatorname{im}(\varphi)$ with finitely many suitable closed disks.) Any $\varphi \in \mathscr{R}\left(M^{2}\right)$ at a distance 0 from $\mathscr{K}\left(M^{2}\right)$ must have compact image, since otherwise there would be a sequence of points in $\operatorname{im}(\varphi)$ no two of which are closer together than some $\varepsilon>0$, and then some $\psi \in \mathscr{K}\left(M^{2}\right)$ would not have compact image. Arguing as before, we see that $\varphi \in \mathscr{K}\left(M^{2}\right)$. Thus $\mathscr{K}\left(M^{2}\right)$ is also closed in $\mathscr{R}\left(M^{2}\right)$, so that $\mathscr{L}\left(M^{2}\right)=\mathscr{K}\left(M^{2}\right)$. If $M^{2}$ is not compact and the compact-open topology is used, or if $M^{2}$ is not complete and the sup-metric topology is used, then $\mathscr{L}\left(M^{2}\right)$ will not be equal to $\mathscr{K}\left(M^{2}\right)$. This can be seen by embedding a half-open interval in $M^{2}$ as a closed subspace and considering retractions onto it and onto closed subintervals of it.

As noted in $\S 1$, in order to prove the theorem, it suffices to prove that the induced map $\Lambda_{*}$ is surjective. Thus let $\Phi:\left(I^{n}, \partial I^{n}\right) \rightarrow\left(\mathscr{L}\left(M^{2}\right), \Lambda\left(u_{0}\right)\right)$ be an element of $\pi_{n}\left(\mathscr{L}\left(M^{2}\right)\right)$, where $u_{0}$ is the basepoint of $M^{2}$. Recall the definition of $\mathscr{S}$ from $\S 2$. For each $\varphi \in \mathscr{L}\left(M^{2}\right)$ such that $\operatorname{im}(\varphi)$ is compact and does not not meet $\partial M^{2}$, let $\mathscr{S}(\varphi)$ denote the set of all $J \in \mathscr{S}$ such that $\operatorname{im}(\varphi)$ is contained in $\operatorname{int}(B(J))$. By Lemma 1 , each $\mathscr{S}(\varphi)$ is nonempty. In both cases of the theorem, use the elbowroom construction of [8, Remark 1.2] to deform $\varphi$ so that $\operatorname{im}(\varphi)$ is disjoint from $\partial M^{2}$ for all $\varphi \in \operatorname{im}(\Phi)$. Let $\mathscr{B}$ be the subspace of $I^{n} \times \mathscr{S}$ consisting of all $(x, J)$ such that $x \in I^{n}$ and $J \in \mathscr{S}(\Phi(x))$. Let $\tau$ be the restriction to $\mathscr{B}$ of the projection of $I^{n} \times \mathscr{S}$ onto $I^{n}$. Choose a fixed $J_{0} \in \mathscr{S}$ such that $u_{0} \in \operatorname{int}\left(B\left(J_{0}\right)\right)$, and let $e: \partial I^{n} \rightarrow \mathscr{B}$ be defined by $e(y)=\left(y, J_{0}\right)$.

We now apply Theorem $\mathrm{M}$ with $X, Y, B, \tau$, and $e$ of the theorem equal to $I^{n}, \partial I^{n}, \mathscr{B}, \tau$, and $e$ as defined in the previous paragraph. In $\S 4$ we verify that the hypotheses of Theorem $M$ are satisfied. Assuming this has been done, we get an extension $e^{*}: I^{n} \rightarrow \mathscr{B}$ such that for each $x \in I^{n}, e^{*}(x) \in$ $\tau^{-1}(x)=\{x\} \times \mathscr{S}(\Phi(x))$. If $\tau_{2}$ denotes the projection onto the second coordinate, then $\tau_{2} \circ e^{*}(x)$ is a canonical member of $\mathscr{S}$ such that the disk it bounds contains $\operatorname{im}(\Phi(x))$ in its interior.

For each $x \in I^{n}$, let $f(x): B\left(\tau_{2} \circ e^{*}(x)\right) \rightarrow B^{2}$ be the homeomorphism which is conformal on the interior and which maps the point ev $\circ \Phi(x)=$ $\Phi(x)\left(u_{0}\right)$ to the origin $(0,0)$. The map $f(x)$ is unique up to a rotation and possibly a reflection (if $M^{2}$ is not orientable), but the construction that follows is independent of rotations and reflections. We can define $\Phi(x)$ piecewise, on $B\left(\tau_{2} \circ e^{*}(x)\right)$ and on the closure of its complement. The map $f(x) \circ \Phi(x) \circ f(x)^{-1}$ is a retraction of $B^{2}$, and thus [8, Theorem 1.1] is homotopic to the constant retraction to $(0,0)$. The homotopy is given by $h_{t} \circ f(x) \circ \Phi(x) \circ f(x)^{-1} \circ h_{t}^{-1} \circ \rho_{t}$, where $\rho_{t}$ projects $A\left(C_{1-t}, C_{1}\right)$ radially to $C_{1-t}$, and $h_{t}$ is a radial homeomorphism of $B^{2}$ onto $\rho_{t}\left(B^{2}\right)$. Thus on 
$B\left(\tau_{2} \circ e^{*}(x)\right)$, add $f(x)^{-1}$ on the left and $f(x)$ on the right to get a homotopy from $\Phi(x)$ to $\Lambda\left(f(x)^{-1}(0,0)\right)$. On $M^{2} \backslash \operatorname{int}\left(B\left(\tau_{2} \circ e^{*}(x)\right)\right)$, use the homotopy $f(x)^{-1} \circ h_{t} \circ f(x) \circ \Phi(x)$. It is easy to see that these two homotopies agree on the curve $\tau_{2} \circ e^{*}(x)$, so together they give a homotopy from $\Phi$ to a map $\Psi^{\circ}$ defined by $\Psi(x)=\Lambda\left(f(x)^{-1}(0,0)\right)=\Lambda \circ \mathrm{ev} \circ \Phi(x)$. Hence $\Phi$ is homotopic to $\Lambda \circ \mathrm{ev} \circ \Phi$, so that $\Lambda_{*}$ is surjective and $\Lambda$ is a weak homotopy equivalence.

4. The hypotheses of Michael's theorem. In $\S 3$, we postponed the verification that the use of Theorem $\mathrm{M}$ is justified. It is easy to see that each $\mathscr{S}(\varphi)$ is homeomorphic to an open subspace of $\mathscr{S}$, and if we prove that $\mathscr{S}$ is $\mathrm{LC}^{n-1}$, then the equi-LC ${ }^{n-1}$ property will follow easily, as in [8, Lemma 2.3]. Using the local metric on $\mathscr{S}$ described below, the proof that $\mathscr{B}$ is an open subspace of $I^{n} \times \mathscr{S}$ is similar to that in [8, Lemma 2.2], and so the map $\tau$ is an open surjection. The remaining hypotheses will be clear from the following lemma.

LEMMA 2. (a) The space $\mathscr{S}$ can be given a metric in which it is complete.

(b) For each $\varphi \in \mathscr{L}\left(M^{2}\right)$ with $\operatorname{im}(\varphi)$ not meeting $\partial M^{2}$, the space $\mathscr{S}(\varphi)$ is contractible (in itself).

(c) The space $\mathscr{S}$ is locally contractible.

Proof. Let $J_{0} \in \mathscr{S}$ and choose a closed neighborhood of $J_{0}$ small enough so that all its elements contain a fixed point $u_{0}$ in their interior. For each $J$ in the neighborhood, consider the unique homeomorphism which maps $B^{2}$ onto $B(J)$, is conformal on the interior, takes the origin to $u_{0}$, and has positive derivative at the origin. (If $M^{2}$ is not orientable, it will be unique up to a reflection.) Let $\mathscr{H}$ denote the space of embeddings $f: C_{1} \rightarrow M^{2}$ such that $f\left(C_{1}\right) \in \mathscr{S}$. (Give $\mathscr{H}$ the topology of uniform convergence.) The remarks above show that a sufficiently small closed neighborhood in $\mathscr{S}$ is homeomorphic to a closed subspace of $\mathscr{H}$. As in [8], $\mathscr{H}$ can be remetrized so as to be complete, so $\mathscr{S}$ can be given a complete metric locally. Also, $\mathscr{S}$ is the continuous image of $\mathscr{H}$ under the obvious projection, so $\mathscr{S}$ is Lindelöf and hence paracompact. Thus $\mathscr{S}$ is metrizable and locally complete, so it can be given a metric which makes it complete [2, pp. 190, 236].

To prove part (b), suppose $J_{0} \in \mathscr{S}(\varphi)$, and let $f$ be any homeomorphism of $B\left(J_{0}\right) \backslash \operatorname{im}(\varphi)$ onto $B^{2}$ minus the origin. For $J \in \mathscr{S}(\varphi)$, let $\alpha$ equal half the supremum of numbers $\beta \in(0,1]$ such that $f^{-1}\left(C_{\beta}\right)$ lies in $B(J)$. Let $K=f^{-1}\left(C_{\alpha}\right)$. For some $r>1$, there is a homeomorphism $g: A(K, J) \rightarrow$ $A\left(C_{1}, C_{r}\right)$ which is conformal on the interior and uniquely determined up to a rotation. The collection of curves $\left\{g^{-1}\left(C_{\beta}\right): 1 \leqq \beta \leqq r\right\}$ defines a deformation in $\mathscr{S}(\varphi)$ from $J$ to $K$. We do this simultaneously for all $J \in \mathscr{S}(\varphi)$ to get a deformation of $\mathscr{S}(\varphi)$ onto the set $\left\{f^{-1}\left(C_{\alpha}\right): 0<\alpha \leqq \frac{1}{2}\right\}$. It is then easy to deform this set in $\mathscr{S}(\varphi)$ to the single curve $f^{-1}\left(C_{1}\right)=J_{0}$. 
For part (c), first prove that $\mathscr{H}$ is locally contractible, using techniques similar to those in [8, Lemma 3.4]. (One can work within a fixed disk in $M^{2}$, extending an embedding to a homeomorphism and using the fact that the space of homeomorphisms of that disk is locally contractible.) Then use the projection of $\mathscr{H}$ onto $\mathscr{S}$ (and the local inverse homeomorphism) to show that $\mathscr{S}$ is locally contractible.

\section{REFERENCES}

1. L. V. Ahlfors and L. Sario, Riemann surfaces, Princeton Math. Series, no. 26, Princeton Univ. Press, Princeton, N.J., 1960. MR 22 \#5729.

2. R. Engelking, Outline of general topology, Biblioteka Mat., Tom 25, PWN, Warsaw, 1965; English transl., North-Holland, Amsterdam, 1968. MR 36 \#4508; MR 37 \#5836.

3. M.-E. Hamstrom and E. Dyer, Regular mappings and the space of homeomorphisms on a 2-manifold, Duke Math. J. 25.(1958), 521-531. MR 20 \#2695.

4. R. Luke and W. K. Mason, The space of homeomorphisms on a compact two-manifold is an absolute neighborhood retract, Trans. Amer. Math. Soc. 164 (1972), 275-285.

5. E. Michael, Continuous selections. II, Ann. of Math. (2) 64 (1956), 562-580. MR 18, 325.

6. R. Nevanlinna, Uniformisierung, Die Grundlehren der math. Wissenschaften, Band 64, Springer-Verlag, Berlin, 1953. MR 15, 208.

7. E. H. Spanier, Algebraic topology, McGraw-Hill, New York, 1966. MR 35 \#1007.

8. N. R. Wagner, The space of retractions of the 2-sphere and the annulus, Trans. Amer. Math. Soc. 158 (1971), 319-329.

Department of Mathematics, University of Texas, El Paso, Texas 79968 